\title{
Primary Outage-Based Resource Allocation Strategies
}

\author{
Bassem Zayen and Aawatif Hayar \\ EURECOM \\ France
}

\section{Introduction}

Cognitive radio (CR) is an emerging technology in wireless technology that uses software-defined radio to aim to the efficient use of the spectrum by exploiting the unused frequency bands at the time and space (J. Mitola, 1999). A look on the state of the art shows that $C R$ research area is very open. A particular problem in the context of CR, when we seek to optimize the secondary system capacity, is to guarantee a quality of service (QoS) to primary users (PUs) and a certain QoS to secondary users (SUs). There is a large number of proposals for all communication layers treating the increase of restrictions to spectrum utilization (Peha, 2005), but the QoS issue still has not been clearly defined. In addition, it is unclear how secondary system opportunism is compatible with the support of QoS for both, CR systems and primary systems. The U.S. Federal Communications Commission (FCC) proposed the concept of "interference temperature" as a way to have unlicensed transmitters sharing licensed bands without causing harmful interference (FCC, 2003). Rather than merely regulate transmitter power at fixed levels, as it has been done in the past, the scheme would have governed transmitter power on a variable basis calculated to limit the energy at victim receivers, where interference actually occurs. As a practical matter, however, the FCC abandoned the interference temperature concept recently (FCC, 2003) due to the fact that it is not a workable concept. While offering attractive promises, CRs face various challenges, starting from defining the fundamental performance limits of this radio technology, in order to achieve the capability of using the spectrum in an opportunistic manner. Specifically, $\mathrm{CR}$ is required to detect spectrum holes in the spectrum band and to determine if the spectrum allocation meets the QoS requirements of different users. This decision can be made by assessing the channel capacity, which is the most important factor for spectrum characterization.

The purpose of this chapter is to present an analysis of the QoS problem along with a proposed solution, while maintaining a limited scope to provide coherency and depth. The QoS problem will be tackled in this work by proposing three resource management strategies based on outage probability. The motivation behind doing so is that, in any case, the PU will not necessarily need all system rate. In fact, the PU will experience the SU's interference, and as long as all his target rate (depending on his QoS) is achieved, he does not care about if he leaves any channel for SUs. In what follows, we adopt this setting and consider a CR network $(\mathrm{CRN})$ in which primary and secondary users both attempt to communicate in a distributed 
way, subject to mutual interference. We propose a CR coordination algorithm that maximizes the CRN secondary rate while keeping the interference to the PU acceptable. Our goal is to realize PU-SU spectrum sharing by optimally allocating SU transmit powers, in order to maximize the total SU throughput under interference and noise impairments, and short term (minimum and peak) power constraints, while preserving the QoS of the primary system. In particular, it is of interest to determine the maximum number of SUs allowed to transmit threshold above which SUs can decide to transmit without affecting the PU's QoS. In such approaches, each user individually makes its decision on its transmit power so as to optimize its contribution to the system throughput. At the core of the concept lies the idea that the interference is more predictable when the network is dense, and consequently the resource allocation problem of a given user becomes more dependent to the average behavior, thus facilitating optimization.

Therefore, we will present and develop in this chapter three resource management strategies based on outage probability. We will derive in a first step a distributed user selection algorithm under a cognitive capacity maximization and outage probability constraints (B. Zayen, M. Haddad, A. Hayar and G.E. Øien, 2008). Specifically, we allow SUs to transmit simultaneously with the PU as long as the interference from the SUs to the PU that transmits on the same band remains within an acceptable range. We impose that SUs may transmit simultaneously with the PU as long as the PU in question does not have his QoS affected in terms of outage probability. The second algorithm investigates multiuser multi-antenna channels using a beamforming strategy (B. Zayen, A. Hayar and G.E. Øien, 2009). The proposed strategy tries to maximize the system throughput and to satisfy the signal-to-interference plus-noise ratio (SINR) constraint, as well as to limit interference to the PU. In the proposed algorithm, SUs are first pre-selected to maximize the per-user sum capacity subject to minimizing the mutual interference. Then, the CR system verifies the outage probability constraint to guarantee QoS for the PU. The third algorithm is based on a game theory tools with the objective to maximize a defined utility function with protection for PUs (B. Zayen, A. Hayar and G. Noubir, 2011). Particularly, we formulate a utility function to reflect the needs of PUs by verifying the outage probability constraint, and the per-user capacity by satisfying the SNIR constraint, as well as to limit interference to PUs. Furthermore, the existence of the Nash equilibrium of the proposed game is established, as well as its uniqueness under some sufficient conditions. Both theoretical and simulation results based on a realistic network setting, for the three presented strategies, will be provided in this chapter and a comparison in terms of CR network (CRN) deployment while maintaining QoS for the PU will be presented.

The chapter is organized as follows. Section 2 will introduce a number of theoretical concepts of importance. It will describe the CRN that will be used throughout this chapter. Section 3 will present the performance metrics used to evaluate the proposed resource allocation algorithms. The following two metrics are considered: PU performance metrics including the primary capacity, the outage probability and the interference outage, and, SU's performance metrics including SU's capacity, SU's sum capacity, the interference power and fairness. In Section 4 and Section 5, we will provide a rather straightforward classification of resource allocation strategies attempting to show the diversity and advantages of these techniques. Two types of resource allocation strategies, centralized strategies, and distributed strategies, are discussed in these two sections, respectively. Section 6 will describe the first distributed resource allocation strategy based on outage probability. Section 7 and Section 8 will present 


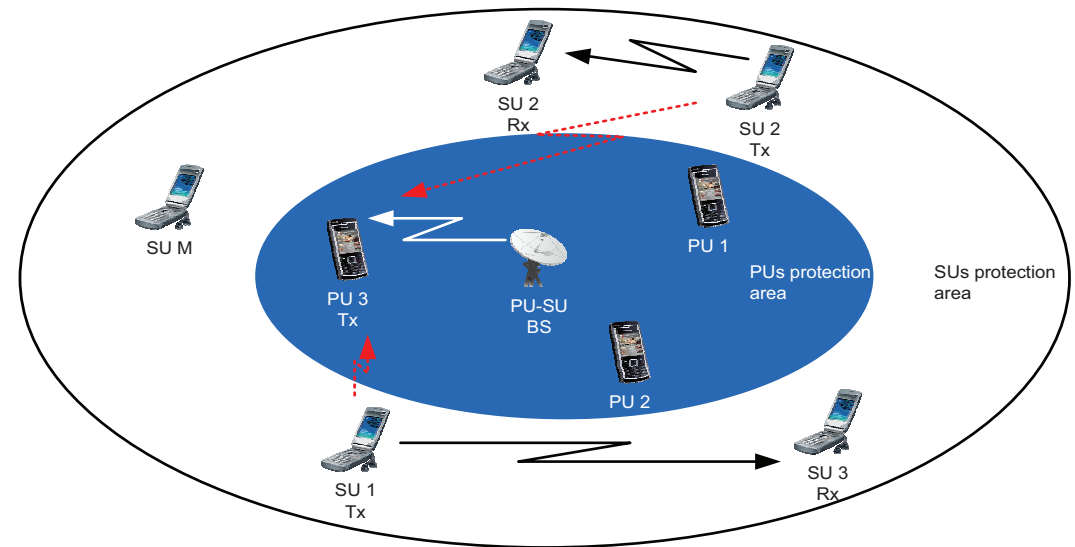

Fig. 1. The cognitive radio network with $N$ primary users and $M$ secondary users attempting to communicate with their respective pairs in an ad-hoc manner during a primary system transmission in downlink mode, subject to mutual interference.

the centralized beamforming-based resource allocation strategy and the one based on game theory, respectively. Section 9 is split in two main subsections. The first subsection will introduce the propagation model that will be used to evaluate the performance of the presented strategy. The second subsection presents simulation results and a comparison of the three user selection strategies presented in this chapter. Section 10 concludes the chapter.

\section{Resource allocation goal}

In order to facilitate the deployment of CR technologies for the secondary usage of spectrum, it is crucial to prove the reliable detection of PUs by SUs. In fact, primary and secondary users can coexist without a degradation of the PU transmission in order to convince regulatory authorities as well as PUs to enable such technologies. Particulary, PU will not necessarily need all that multi-rate system. In fact, the PU will experience the SU's interference, and as long as all his target rate (depending on his QoS) to be achieved, he does not care about if he leaves any channel for SUs. In what follows, we adopt this setting and consider a CRN in which primary and secondary users both attempt to communicate, subject to mutual interference. This is the main goal of the study in the second part of this chapter.

We consider here a wireless CRN with a collection of users randomly distributed over the geographical area considered. Users can be both transmitters and receivers. By virtue of a scheduling protocol, N PUs and M pairs of SUs are simultaneously selected from these users to communicate at a given time instant, while others remain silent. We will consider in our analysis both downlink and uplink scenarios. We show in Figure 1 the downlink scenario as an example. In this scenario, we assume that a BS transmits to its user which has the highest channel power gain. In the uplink scenario, its users transmit to the network's BS. We introduce also in the presented figure the interference channel gain between SUs transmitters and PU transmitter. Provided that no significant scatterers are presented in the area, the channel gains between any pair of users are assumed i.i.d. in the two proposed scenarios and they depend on the position of the users in the two-dimensional plane. Each PU is allocated 
with a unique resource slot so that it transmits in an orthogonal manner with respect to other PUs within its coverage area, i.e. no interference between different PUs like in the Orthogonal Frequency-Division Multiple Access-based (OFDMA-based systems). All details about the CRN parameters and the propagation model in the downlink and the uplink mode will be given in Section 9. In this CRN, we will consider only the case when we have one PU and $M$ pairs of SUs.

In order to facilitate the problem formulation of the resource allocation problem, we state the following notations:

- the PU is indexed by $p u$,

- the index of SU $m$ lies between 1 and $M$,

- $h_{l, m}$ denotes the channel gain from SU $l$ to the desired user $m$,

- the data destined from SU $m$ is transmitted with power $p_{m}$ and a maximum power $P_{\max }$,

- $h_{p u, m}$ denotes the channel gain from the PU indexed by $p u$ to the desired user $m$,

- $h_{p u, p u}$ denotes the channel gain between the BS and the PU,

- the data destined from the primary system is transmitted with power $p_{p u}$.

In the coverage area of the primary system, there is an interference boundary within which no SUs can communicate in an ad-hoc manner. Thus, as can be seen in Figures 1, for the impairment experienced by the primary system to be as small as possible, a SU must be able to detect very reliably whether it is far enough away from a primary base station, i.e., in the area of possible CR operation. Under these schemes, we allow SUs to transmit simultaneously to the PU as long as the interference from the SUs to the PU that transmits on the same band remains within an acceptable range. Specifically, we impose that SUs may transmit simultaneously with the PU as long as the PU in question does not have its QoS affected. Based on PU channel statistics, we determine a QoS bound to ensure a protection to the PU. To compute this bound, we will use the outage probability.

For system design purposes we will need to define metrics that will guide our development. These metrics need to be sufficiently broad, so that a realistic system can be designed through an optimization of all the metrics that we define. This would therefore need to include metrics to measure the performance of the CRN. These metrics will be given in the following section.

\section{Resource allocation metrics}

Algorithms that aid reliable resource management need to be verified and their performance has to be quantified by some metrics. In this section, we will present the performance metrics used to evaluate the proposed resource allocation algorithms.

\subsection{Primary users performance metrics}

Primary Capacity In most of resource allocation strategies, algorithms must ensure that the transmission rate of PU is no bigger than the primary capacity for most of the time. The PU instantaneous capacity is give by 


$$
C_{p u}=\log _{2}\left(1+\frac{p_{p u}\left|h_{p u, p u}\right|^{2}}{\sum_{m=1}^{M} p_{m}\left|h_{p u, m}\right|^{2}+\sigma^{2}}\right)
$$

where $\sigma^{2}$ is the ambient noise variance. Clearly, the primary capacity is directly related to the PU transmission as well as the SUs transmission.

Outage Probability The notion of information outage probability, defined as the probability that the instantaneous mutual information of the channel is below the transmitted code rate, was introduced in (L. H. Ozarow, S. Shamai and A.D. Wyner, 1994). Accordingly, the outage probability can be written as:

$$
P_{\text {out }}(R)=P\{I(\mathbf{x} ; \mathbf{y}) \leq R\}
$$

where $I(\mathbf{x} ; \mathbf{y})$ is the mutual information of the channel between the transmitted vector $\mathbf{x}$ and the received vector $\mathbf{y}$, and $R$ is the target data rate in bits/s/Hz. Reliable communication can therefore be achieved when the mutual information of the channel is strong enough to support the target rate $R$. Thus, a $m$-th cognitive transmitter can adapt its transmit power $p_{m}$ within the range of $\left[0 ; P_{\max }\right]$ to fulfill the following two basic goals:

- Self-goal: Trying to transmit as much information for itself as possible,

- Moral-goal: Maintaining the PU's outage probability unaffected.

The outage probability can be rewritten as:

$$
P_{\text {out }}=\operatorname{Prob}\left\{\mathrm{C}_{p u} \leq R_{p u}\right\}
$$

where $R_{p u}$ is the PU transmitted data rate. The information about the outage failure can be carried out by a band manager that mediates between the primary and secondary users, or can be directly fed back from the PU to the secondary transmitters through collaboration and exchange of the CSI between the primary and secondary users.

Interference Outage The CR specific metrics relate to how well the CR is able to avoid PU and the efficiency in using available spectrum. This will require a model for PU dynamics, such as disappearance and reappearance time intervals, the amount of spectrum being used and the strength and location of the PU. In addition to the primary capacity and the outage probability, we define the interference outage meaning when the power of interference at a receiver PU exceeds a pre-defined absolute limit. Let $q$ be this absolute limit (i.e. the maximum outage probability).

\subsection{Secondary users performance metrics}

Secondary User's Capacity By making SUs access the primary system spectrum, the $m$-th SU experiences interference from the PU and all neighboring co-channel SU links that transmit on the same band. Accordingly, the $m$-th SU instantaneous capacity is given by:

$$
C_{m}=\log _{2}\left(1+\operatorname{SINR}_{m}\right)
$$


where

$$
\operatorname{SINR}_{m}=\frac{p_{m}\left|h_{m, m}\right|^{2}}{\sum_{\substack{l=1 \\ l \neq m}}^{M} p_{l}\left|h_{l, m}\right|^{2}+p_{p u}\left|h_{p u, m}\right|^{2}+\sigma^{2}}
$$

Secondary User's Sum Capacity SUs need to recognize their communication environment and to adjust the parameters of their communication scheme in order to maximize the per-user cognitive capacity, expressed as

$$
C_{s u}=\sum_{m=1}^{M} C_{m}
$$

while minimizing the interference to the PUs, in a distributed fashion. The sum here is made over the M SUs allowed to transmit. Moreover, we assume that the coherence time is sufficiently large so that the channel stays constant over each scheduling period length. We also assume that SUs know the channel state information (CSI) of their own links, but have no information on the channel conditions of other SUs.

Interference Power No interference cancelation capability is considered in our study. Power control is used for SUs both in an effort to preserve power and to limit interference and fading effects. The interference power (Intf) is given by:

$$
\operatorname{Intf}_{m}=\sum_{\substack{l=1 \\ l \neq m}}^{M} p_{l}\left|h_{l, m}\right|^{2}+p_{p u}\left|h_{p u, m}\right|^{2}+\sigma^{2}
$$

Combining (5) and (7), we define the SINR as a function of Intf:

$$
\operatorname{SINR}_{m}=\frac{p_{m}\left|h_{m, m}\right|^{2}}{\operatorname{Intf}_{m}}
$$

and

$$
p_{m}=\frac{\operatorname{SINR}_{m} \operatorname{Intf}_{m}}{\left|h_{m, m}\right|^{2}}
$$

\section{Centralized resource allocation strategies}

In the centralized mode, the resource allocation system would require a central controller and information about all users and channels. The centralized resource allocation have been the main focus of some research efforts in CRNs. We will provide in this section some solutions to this issue that have been proposed in the literature.

The authors in (L. Qian, X. Li, J. Attia and Z. Gajic, 2007) derived a centralized power control method for the CRN to maximize the energy efficiency of the SUs and guarantee the QoS of both the PUs and the SUs. The feasibility condition was derived in (L. Qian, X. Li, J. Attia and Z. Gajic, 2007) and a joint power control and admission control procedure was suggested such that the priority of the PUs is ensured all the time. However, in (L. Qian, X. Li, J. Attia and Z. Gajic, 2007) only one CRN was considered. 
In (Y. Xing, C.N. Mathur, M.A. Haleem, R. Chandramouli and K.P. Subbalakshmi, 2007), the authors considered spectrum sharing among a group of spread spectrum users with a constraint on the total interference temperature at a particular measurement point, and a QoS constraint for each secondary link. An optimization solution of this problem was proposed in (Y. Xing, C.N. Mathur, M.A. Haleem, R. Chandramouli and K.P. Subbalakshmi, 2007) by using a game theory method. Specifically, the authors defined the secondary spectrum sharing problem as a potential game which takes different priority classes into consideration. Firstly, this game is solved through sequential play. Then a learning automata algorithm is introduced which only requires a feedback of the utility value. The same idea was proposed in (J. Huang, R.A. Berry and M.L. Honig, 2006), where the authors study a centralized auction mechanisms to allocate the received powers. They consider an objective function of maximizing utility which is a function of SINR. In (E. Jorswieck and R. Mochaourab, 2010) the authors tried to solve the centralized resource allocation problem by including a beamforming strategy. In this work, the primary systems are assumed to tolerate an amount of interference originating from secondary systems. This amount of interference is controlled by a pricing mechanism that penalizes the secondary systems in proportion to the interference they produce on the PUs.

Two centralized optimization frameworks were proposed in (L. Akter and B. Natarajan, 2009) in order to solve for the optimal resource management strategies. In the first framework, authors determine the minimum transmit power that SUs should employ in order to maintain a certain SINR and use that result to calculate the optimal rate allocation strategy across channels. In the second framework, both transmit power and rate per channel are simultaneously optimized with the help of a bi-objective problem formulation.

Though there have been ample research efforts on centralized resource management in CRNs, there is still a lack of a complete framework that considers QoS for SUs as well as resource management in a fair manner. One of the objective in this chapter is to take a step towards such a solution.

In a realistic network, centralized system coordination is hard to implement, especially in fast fading environments and in particular if there is no fixed infrastructure for SUs, i.e., no back-haul network over which overhead can be transmitted between users. In fact, centralized channel state information for a dense network involves immense signaling overhead and will not allow the extraction of diversity gains in fast-fading channel components. To alleviate this problem, distributed methods were proposed in the literature where SUs can communicate without PU knowledge.

\section{Distributed resource allocation strategies}

In the centralized case, the need may exist, as mentioned above, for the perfect knowledge of all channel and interference state conditions for all nodes in the network. To circumvent this problem, the design of so-called distributed resource allocation techniques is crucial. Distributed optimization refers to the ability for each user to manage its local resources (e.g. rate and power control, user scheduling) based only on locally observable channel conditions such as the channel gain between the access point and a chosen user, and possibly locally measured noise and interference. 
A number of distributed resource allocation strategies for CRNs have been proposed in literature. In addition to the two centralized frameworks presented in last section, the authors in (L. Akter and B. Natarajan, 2009) designed a distributed suboptimal joint coordination and power control mechanism to allocate transmit powers to SUs. A lower bound on SINR is used as a QoS constraint for SUs. In (N. Nie and C. Comaniciu, 2005), the authors propose a game theoretic framework to analyze the behavior of CRs for distributed adaptive channel allocation. They define two different objective functions for the spectrum sharing games, which capture the utility of selfish users and cooperative users, respectively. The channel allocation problem is modeled in (N. Nie and C. Comaniciu, 2005) to a potential game which converges to a deterministic Nash equilibrium channel allocation point. Game theory was applied in (F. Wang, M. Krunz and S. Cui, 2008) to develop a distributed power allocation algorithm. In this work, each user maximizes its own utility function (which includes a pricing term) by performing a single-user price-based water-filling. However, in (F. Wang, M. Krunz and S. Cui, 2008), coexistence of multiple SUs in a channel has not been considered. Also, the QoS requirement of SUs has been ignored. In (Y. Wu and D.H.K. Tsang, 2009), the authors studied the distributed multi-channel power allocation for spectrum sharing CRNs with QoS guarantee. They formulate the problem as a noncooperative game with coupled strategy space to address both the co-channel interference among SUs and the interference temperature regulation imposed by primary systems.

The authors in (P. Cheng, Z. Zhang, H. Huang and P. Qiu, 2008) presented a general analytical framework, in which SU's rate, frequency, and power resource can be jointly optimized under the interference temperature constraints. This framework was used to design an optimal distributed resource allocation algorithm with low polynomial time complexities in multiuser broadband CRNs. In (J. Huang, Z. Han, M. Chiang and V. Poor, 2007), the authors focus on designing distributed resource allocation algorithms for cooperative networks. They proposed two share auction mechanisms, the SNR auction and the power auction, to distributively coordinate the relay power allocation among users. The authors in (J. Huang, Z. Han, M. Chiang and V. Poor, 2007) demonstrate that the SNR auction achieves the fair allocation, while the power auction achieves the efficient allocation.

In (D. Schmidt, C. Shi, R. Berry, M. Honig and W. Utschick, 2009), the authors propose a distributed resource allocation scheme where SUs are penalized for interfering on the primary systems. The penalty is proportional to the interference rate produced from the secondary transmitter to each PU. This mechanism is referred to as pricing and is interpreted as introducing the effect of disturbance created from a user as a penalty measure in his utility function. In this means, the secondary transmitters can be controlled to choose their transmission strategies satisfying soft interference constraints on the PUs. In (J. S. Pang, G. Scutari, D. Palomar and F. Facchinei, 2010), this model of exogenous prices is used to analyze a noncooperative game between the SUs. Distributed algorithms are provided that iteratively modify the prices weights and eventually reach the Nash equilibrium that satisfies the interference temperature constraints.

\section{Distributed strategy}

In the current study, we adopt a QoS guarantee to the PU by means of an outage constraint. This knowledge can be obtained with a centralized mode where the resource allocation system 
would require information from a third party (i.e. central database maintained by regulator or another authorized entity) to schedule SUs coming. This is the case of the user selection strategy presented in (M. Haddad, A. Hayar and G.E. Øien, 2008). In fact, to compute the $P_{\text {out }}$, the CR system requires knowledge of the PU and SUs channels. To alleviate this problem, we propose in this chapter a distributed method where SUs can get rid of PU knowledge. In this distributed framework, the information about the outage failure can be computed without exchange of information between the primary and secondary users. In this section, we will present in a first step a reformulation of the outage probability that will be used throughout the development of the proposed user selection strategy. Then, we will present the optimization problem of this strategy.

\subsection{Binary power control policy}

One basic assumption throughout this part is that a SU can vary its transmit power, under short term (minimum and peak) power constraints, in order to maximize the cognitive capacity, while maintaining a QoS guarantee to the PU. For the first proposed distributed resource allocation algorithm, we will use a binary power control (nodes transmitting at maximum power $P_{\max }$ or being silent).

The idea of the binary "on" / "off" power control is simple, as well as yielding quasi-optimal results in a number of cases (A. Gjendemsjø, D. Gesbert, G. E. Øien and S.G. Kiani, 2007). As such, it constitutes a promising tool for making spectrum sharing a reality. Besides complexity reduction, an important additional benefit of binary power control is to allow distributed optimization. With binary power constraints, power control reduces to deciding if links should be "on" or "off". The power $p_{m}$ of the $m$-th SU transmitter is selected from the binary set $\left\{0, P_{\max }\right\}$. It is intuitively clear that if the cross-gain is sufficiently low, then all links should be "on".

The key idea within the iterative algorithm used in the development of the proposed distributed user selection algorithm is, as in (Kiani \& Gesbert, 2006), to subsequently limit $p_{m}$ to $\left\{0, P_{\max }\right\}$, i.e., to switch "off" transmission in SUs' links which do not contribute enough capacity to outweigh the interference degradation caused by them to the rest of the network. The authors in (M. Haddad, A. Hayar and G.E. Øien, 2008) propose an adaptation of the distributed algorithm which allows a subset of controlled size $\tilde{M}$ of the total number of SUs $M$ to transmit simultaneously on the same sub-band. We will give in this section a summary of the presented method in (M. Haddad, A. Hayar and G.E. Øien, 2008).

At High SINR Regime Assuming all SUs to be in "on" condition for the mentioned CRN, at high SINR regime, we have dense environment with more users within smaller geometrical area and hence a SU requires higher threshold to be active. Assuming that $1+$ SINR $=$ SINR holds, the signal-to-interference ratio (SIR) threshold for high region comes out to be,

$$
\operatorname{SIR}_{m}=\frac{p_{m}\left|h_{m, m}\right|^{2}}{p_{p u}\left|h_{p u, m}\right|^{2}+\sum_{\substack{k \in \Psi \\ k \neq m}} p_{k}\left|h_{k, m}\right|^{2}}>e=2.718281 \ldots
$$


At Low SINR Regime By definition in the low SINR region $\ln (1+x) \simeq x$ holds with good accuracy, and binary power control is always optimal. The active user threshold at low SINR region is expressed as,

$$
\operatorname{SIR}_{m}>1
$$

Detailed derivations of the two threshold at high and low SINR are given in (M. Haddad, A. Hayar and G.E. Øien, 2008). Results given in (10) and (11) confirm, as intuition would expect, that SUs under better SINR conditions would transmit only above a higher threshold than in the low SINR regime.

\subsection{Outage probability constraint}

To proceed further with the analysis of the distributed strategy and for the sake of emphasis, we introduce the PU average channel gain estimate $G_{p u}$ based on the following decomposition:

$$
h_{p u, p u} \triangleq G_{p u} * h_{p u, p u}^{\prime}
$$

where $h_{p u, p u}^{\prime}$ is the random component of channel gain and represents the normalized channel impulse response tap (A. Gjendemsjø, D. Gesbert, G. E. Øien and S.G. Kiani, 2007). This gives us the following PU outage probability expression:

$$
P_{\text {out }}=\operatorname{Prob}\left\{\log _{2}\left(1+\frac{p_{p u} G_{p u}^{2}\left|h_{p u, p u}^{\prime}\right|^{2}}{\sum_{m=1}^{M} p_{m}\left|h_{p u, m}\right|^{2}+\sigma^{2}}\right) \leq R_{p u}\right\}
$$

Let $\tilde{M}_{d}$ be the maximum number of SUs allowed to transmit using the distributed method and $G_{s u}$ be the SU average channel gain estimate. If we insert these two parameters in (13), we obtain

$$
\begin{aligned}
P_{\text {out }} & \simeq \operatorname{Prob}\left\{\frac{p_{p u} G_{p u}^{2}\left|h_{p u, p u}^{\prime}\right|^{2}}{G_{s u}^{2} \sum_{m=1}^{\tilde{M}_{d}} p_{m}+\sigma^{2}} \leq 2^{R_{p u}}-1\right\} \leq q \\
& \simeq \operatorname{Prob}\left\{\left|h_{p u, p u}^{\prime}\right|^{2} \leq\left(2^{R_{p u}}-1\right)\left(\frac{\tilde{M}_{d} G_{s u}^{2} P_{\text {max }}+\sigma^{2}}{G_{p u}^{2} p_{p u}}\right)\right\} \leq q
\end{aligned}
$$

From now on we assume for simplicity of analysis that the channel gains are i.i.d Rayleigh distributed. However, the results can be immediately translated into results for any other channel model by replacing the appropriate probability distribution function. Continuing from (14), we have:

$$
P_{\text {out }} \simeq \int_{0}^{\left(2^{R_{p u}}-1\right)\left(\frac{\tilde{M}_{d} G_{s u}^{2} P_{\max }+\sigma^{2}}{G_{p u}^{2} p_{p u}}\right)} \exp (-t) d t \leq q
$$


Finally, we get the following outage constraint:

$$
P_{\text {out }} \simeq 1-\exp \left[-\left(2^{R_{p u}}-1\right)\left(\frac{\tilde{M}_{d} G_{s u}^{2} P_{\max }+\sigma^{2}}{G_{p u}^{2} p_{p u}}\right)\right] \leq q
$$

and the maximum number $\tilde{M}_{d}$ of active "on" SUs that transmit with $P_{\max }$ is given by

$$
0 \leq \tilde{M}_{d} \leq \frac{-\log (1-q)}{\left(2^{R_{p u}}-1\right)} \cdot \frac{G_{p u}^{2} p_{p u}}{G_{s u}^{2} P_{\max }}-\frac{\sigma^{2}}{G_{s u}^{2} P_{\max }}
$$

By writing SNR $=\frac{G_{S u}^{2} P_{\max }}{\sigma^{2}}$, equation (17) can be expressed as:

$$
0 \leq \tilde{M}_{d} \leq \frac{-\log (1-q)}{\left(2^{R_{p u}}-1\right)} \cdot \frac{G_{p u}^{2} p_{p u}}{G_{s u}^{2} P_{\max }}-\frac{1}{\mathrm{SNR}}=\tilde{M}_{\text {theory }}
$$

where $\tilde{M}_{\text {theory }}$ is the theoretic maximum number of SUs allowed to transmit. The LHS in (18) prevents from obtaining a negative number of users when the SNR decreases significantly. The formula in (18) points out that the number of SUs allowed to transmit increases as their SNR increases.

\subsection{Optimization problem}

The SUs offer the opportunity to improve the system throughput by detecting the PU activity and adapting their transmissions accordingly while avoiding the interference to the PU by satisfying the QoS constraint on outage. We present in this subsection a distributed user selection strategy using the binary power allocation policy given in Section 6.1. The proposed strategy tries to limit the number of SUs interfering with the PU so as to guarantee the QoS for the primary system. Specifically, a SU will be deactivated if its action results in an increase in the cognitive capacity of SUs or if its transmission violates the PU outage constraint. The optimization problem can therefore be expressed as follows:

$$
\text { Find }\left.p_{m}\right|_{m=1, \ldots, M}=\arg \max _{p_{m}} C_{s u}
$$

subject to:

$$
\left\{\begin{array}{l}
p_{m} \in\left\{0, P_{\max }\right\}, \quad \text { for } m=1, \ldots, M \\
0 \leq \tilde{M}_{d} \leq \frac{-\log (1-q)}{\left(2^{R_{p u}}-1\right)} \cdot \frac{G_{p u}^{2} p_{p u}}{G_{s u}^{2} P_{\max }}-\frac{1}{\mathrm{SNR}}
\end{array}\right.
$$

where $\tilde{M}_{d}$ is the maximum number of SUs allowed to transmit using the distributed algorithm and $q$ the maximum outage probability. As we can see from (20), the CR system does not require knowledge about the PU and SUs channels in the sense that it decides distributively to either SU transmit data or stay silent over the channel coherence time depending on the specified $P_{\text {out }}$ threshold $(q)$. On the other hand, the optimization problem presented in (M. 


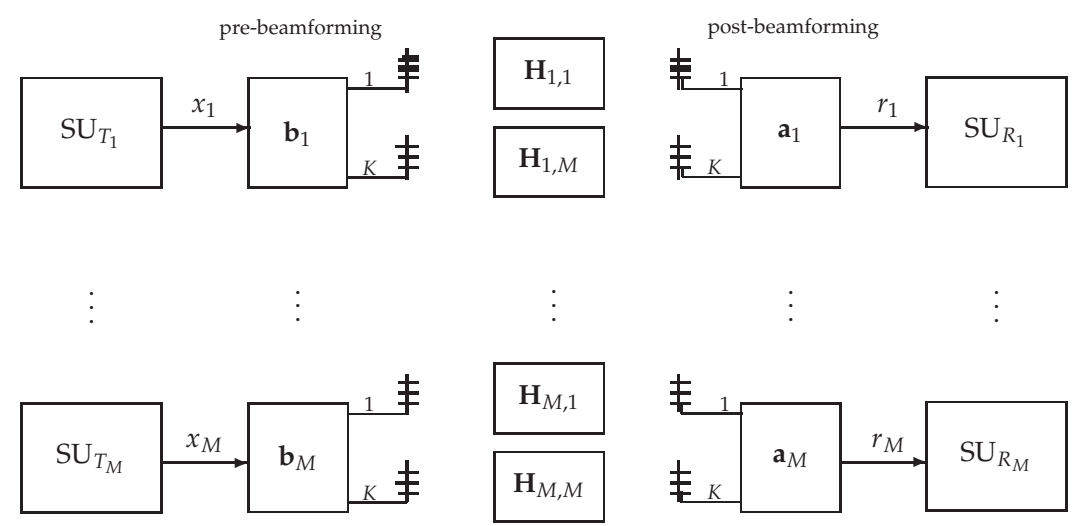

Fig. 2. Multiple transmit and receive secondary users system structure.

Haddad, A. Hayar and G.E. Øien, 2008) requires all $h_{m, p u}$ and $h_{p u, p u}$ data to compute the outage probability and to select then the SUs able to transmit without affecting the PUs' QoS.

An iterative approach is adopted throughout this algorithm. The algorithm is first initialized with a zero power allocation vector. Each SU simultaneously measures his SIR; depending on whether the SU is on high or low average SINR, he remains active or inactive during the next time slot based on (10) and (11), respectively. Similarly, at every iteration of the Monte Carlo simulation, $\tilde{M}_{d}$ is evaluated for the SU in question based on the average channel gains $G_{s u}$ and $G_{p u}$ estimation. The algorithm is run until the outage probability stabilizes. The last SU entering in the system is removed from the transmission.

\section{Centralized beamforming-based resource allocation strategy}

In this section, we will present the design of the transmit and receive beamvectors. In fact, beamvector associated with each SU is determined by optimizing a certain criterion to reach a specific purpose such as maximizing the throughput or minimizing the interference. In the literature, depending on the objective function and the constraints, the beamforming optimization problems can be divided into two classes. One is the SINR balancing problem (M. Schubert and H. Boche, 2004), i.e., maximizing the sum SINR among all the users. The other one is the power minimization problem with SINR constraints (M. Schubert and H. Boche, 2004), i.e., minimizing some power function with SINR constraints. In this work, we adopt the first class combined with an outage probability constraint, i. e., we will maximize the per-user sum capacity subject to minimize the mutual interference. The goal here is to choose for each user who has the best equivalent channel created by the multiple antennas and beamforming where resources are allocated. In this section, we introduce the power constraints to compute beamvectors. Then, we present the outage probability constrain. Finally, we present the optimization problem of the proposed strategy.

\subsection{Secondary users MIMO system}

In this subsection, we will describe the SU MIMO system model and multiuser diversity scheme that we are considering in this chapter, and discuss the primary and secondary users 
metrics when we use a SU MIMO system. We will reformulate the resource allocations metrics given in Section 3 when we use a SU single-input-single-output (SISO) system.

The proposed system in this chapter consists of multiple transmit/receive SU links randomly distributed over the geographical area considered. MIMO systems have great potential to enhance the capacity in the framework of wireless cellular networks (E.A. Jorswieck and H. Boche, 2004). Multiple antennas can for example be deployed at a cognitive BS. Many wireless network standards provision the use of transmit antenna arrays. Using baseband beamforming, it is possible to steer energy in the direction of the intended users, whose channels can often be accurately estimated (A. Tarighat, M. Sadek and A.H. Sayed, 2005; E.A. Jorswieck and H. Boche, 2004). Beamforming has been also exploited as a strategy that can serve many users at similar throughput. Moreover, beamforming has the advantage of limiting interference. Thus, we are interested in transmit beamforming schemes for cognitive transmission. For this purpose, we utilize joint beamforming that implies an extension to the transmitter side of classical receive beamforming (P. Viswanath, D. Tse and R. Laroia, 2004).

The SU system structure is based on beamforming at both the transmitter ( $K$ antennas) and the receiver ( $K$ antennas) for each SU link as given in Fig. 2. The number of secondary transmitters $\left(\mathrm{SU}_{T}\right)$ is equal to $M$, and is equal to the number of secondary receivers $\left(\mathrm{SU}_{R}\right)$. Assuming that many scatterers are located around the transmitter and receivers, the channel coefficient matrix $\mathbf{H}_{r t}$ (the channel between the $t$-th transmit SU and the $r$-th receive SU) exhibits flat fading. The channel gain vector $\mathbf{h}_{p u, m}$ from the PU indexed by $p u$ to a desired SU $m$ ( $m$ between 1 and $M$ ) is given by:

$$
\mathbf{h}_{p u, m}=\left[h_{p u, m_{1}} \ldots h_{p u, m_{K}}\right]^{T}
$$

where the channel gains are assumed i.i.d. random variables. We consider that the channels between different users are independent. We then set the received signal of the $m$-th user as follows (the index of SUs $m$ lies between 1 and $M$ ):

$$
\mathbf{y}_{m}=\mathbf{H}_{m, m} \mathbf{s}_{m}+\sum_{l=1, l \neq m}^{M} \mathbf{H}_{m, l} \mathbf{s}_{l}+\mathbf{h}_{p u, m} x_{p u}+\mathbf{n}_{m}, \quad m=1, \ldots, M
$$

with $\mathbf{n}_{m}$ of size $K \times 1$ being zero-mean i.i.d. Gaussian noise with power $\sigma_{m}^{2}$, and $K$ being the number of antennas. $\mathbf{s}_{m}$ is the transmit vector of size $K \times 1$ for the $m$-th SU and $x_{p u}$ being the transmit sample sent from PU. $\mathbf{y}_{m}$ is the receive vector of size $K \times 1$. $\mathbf{H}_{m, m}(K \times K$ matrix $)$ is the channel between the $m$-th $\mathrm{SU}_{T}$ and the $m$-th $\mathrm{SU}_{R}$ and $\mathbf{H}_{m, l}(l=1, \ldots, m-1, m+1, \ldots, M)$ are channel matrices between the other SUs, referred to as the interference channel matrices.

Here, a joint beamforming approach is proposed for the SU system, that is, all the transmitters and receivers exploit a beamforming architecture (E.A. Jorswieck and H. Boche, 2004). The transmission scheme is characterized by the power allocation (eigenvalues of the transmit covariance matrix) and the orientation (eigenvectors of the transmit covariance matrix) (Tse, 2004). This yields

$$
\mathbf{s}_{m}=\mathbf{b}_{m} x_{m}, \quad m=1, \ldots, M
$$

where $\mathbf{b}_{m}$ is the pre-beamforming vector and $x_{m}$ is the transmit sample for $m$ between 1 and $M$. The output of the $m$-th receiver beamformer is: 


$$
\begin{aligned}
r_{m} & =\mathbf{a}_{m}^{H} \mathbf{y}_{m} \\
& =\mathbf{a}_{m}^{H} \mathbf{H}_{m, m} \mathbf{b}_{m} x_{m}+\mathbf{a}_{m}^{H} \sum_{l=1, l \neq m}^{M} \mathbf{H}_{m, l} \mathbf{b}_{l} x_{l}+\mathbf{a}_{m}^{H} \mathbf{h}_{p u, m} x_{p u}+\mathbf{a}_{m}^{H} \mathbf{n}_{m}
\end{aligned}
$$

where $\mathbf{a}_{m}$ is the post-beamforming vector at the receive SUs. $\Phi_{m}=E\left\{\mathbf{n}_{m} \mathbf{n}_{m}^{H}\right\}$ is the associated covariance matrix. The SINR defined in (5) at the $m$-th SU can be rewritten as:

$$
\begin{aligned}
\operatorname{SINR}_{m} & =\frac{E\left\{\left|\mathbf{a}_{m}^{H} \mathbf{H}_{m, m} \mathbf{b}_{m} x_{m}\right|^{2}\right\}}{E\left\{\sum_{l=1, l \neq m}^{M}\left|\mathbf{a}_{m}^{H} \mathbf{H}_{m, l} \mathbf{b}_{l} x_{l}\right|^{2}\right\}+E\left\{\left|\mathbf{a}_{m}^{H} \mathbf{h}_{p u, m} x_{p u}\right|^{2}\right\}+E\left\{\left|\mathbf{a}_{m}^{H} \mathbf{n}_{m}\right|^{2}\right\}} \\
& =\frac{\left|\mathbf{a}_{m}^{H} \mathbf{H}_{m m} \mathbf{b}_{m}\right|^{2}}{\left|\mathbf{a}_{m}^{H} \mathbf{h}_{p u, m}\right|^{2}+\sum_{l=1, l \neq m}^{M}\left|\mathbf{a}_{m}^{H} \mathbf{H}_{m, l} \mathbf{b}_{l}\right|^{2}+\mathbf{a}_{m}^{H} \mathbf{R}_{m} \mathbf{a}_{m}}
\end{aligned}
$$

and the capacity of PU is given in this context by:

$$
\mathrm{C}_{p u}=\log _{2}\left(1+\frac{p_{p u}\left|h_{p u, p u}\right|^{2}}{\sum_{m=1}^{M}\left|\mathbf{h}_{p u, m} \mathbf{h}_{p u, m}{ }^{H}\right|\left|\mathbf{b}_{m}\right|^{2}+\sigma^{2}}\right)
$$

An efficient transmit beamforming technique combined with user selection will be proposed in the following section by optimizing a certain problem.

\subsection{Power constraints}

To compute the beamvectors, we consider just the SU MIMO system. The reason for this is that the interference among PU is nulled in SINR equation (i.e. $\mathbf{a}_{m}^{H} \mathbf{h}_{p u, m}=0$ ). In fact, we propose an algorithm that can minimize the interference between cognitive users. SUs are first pre-selected so as to maximize the per-user sum capacity, and then, the PU verifies the outage probability constraint and a number of SUs are selected from those pre-selected SUs. Specifically, beamvectors are selected such that they satisfy the interference free condition $\mathbf{a}_{m}^{H} \mathbf{h}_{p u, m}=0$. If we consider this condition, the SINR at the $m$-th SU can then be written as:

$$
\mathrm{SINR}_{m}=\frac{\left(\mathbf{a}_{m}^{H} \mathbf{H}_{m, m} \mathbf{b}_{m}\right)^{H}\left(\mathbf{a}_{m}^{H} \mathbf{H}_{m, m} \mathbf{b}_{m}\right)}{\mathbf{a}_{m}^{H}\left(\Phi_{m}+\sum_{l=1, l \neq m}^{M} \mathbf{H}_{m, l} \mathbf{b}_{l} \mathbf{b}_{l}^{H} \mathbf{H}_{m, l}^{H}\right) \mathbf{a}_{m}}
$$

We define the total interference plus noise covariance matrix at the $m$-th SU as:

$$
\mathbf{R}_{m}=\Phi_{m}+\sum_{l=1, l \neq m}^{M} \mathbf{H}_{m, l} \mathbf{b}_{l} \mathbf{b}_{l}^{H} \mathbf{H}_{m, l}^{H}
$$


Therefore, the SINR at the $m$-th SU can be formulated as follows:

$$
\begin{aligned}
\operatorname{SINR}_{m} & =\frac{\left(\mathbf{a}_{m}^{H} \mathbf{H}_{m, m} \mathbf{b}_{m}\right)^{H}\left(\mathbf{a}_{m}^{H} \mathbf{H}_{m, m} \mathbf{b}_{m}\right)}{\mathbf{a}_{m}^{H} \mathbf{R}_{m} \mathbf{a}_{m}} \\
& =\mathbf{b}_{m}^{H} \mathbf{H}_{m, m} \mathbf{R}_{m}^{-1} \mathbf{H}_{m, m}^{H} \mathbf{b}_{m}
\end{aligned}
$$

From (29), the post-beamforming vector can be expressed as follows:

$$
\mathbf{a}_{m}=\mathbf{R}_{m}^{-1} \mathbf{H}_{m, m} \mathbf{b}_{m}
$$

This gives us the following maximization of SINR at the $m$-th SU:

$$
\mathbf{b}_{m}^{H} \mathbf{H}_{m, m}^{H} \mathbf{R}_{m}^{-1} \mathbf{H}_{m, m} \mathbf{b}_{m} \leq \lambda_{\max }(m) p_{m}=\left.\mathrm{SINR}_{m}\right|_{\text {max }}
$$

where $\lambda_{\max }(m)$ is the maximum eigenvalue of $\mathbf{H}_{m, m}^{H} \mathbf{R}_{m}^{-1} \mathbf{H}_{m, m}$ and $p_{m}=\mathbf{b}_{m}^{H} \mathbf{b}_{m}$. For beamforming, the transmitted power through all the SUs for the $m$-th SU is proportional to $\left\|\mathbf{b}_{m}\right\|^{2}$. The design goal is to find the optimum transmit weight vector subject to a carrier power constraint. We consider the power allocation problem corresponding to the distribution of all the available power at the transmitter among all SUs, when the data destined from SU $m$ is transmitted with a maximum power $P_{\max }$. This per-user power constraint is given by:

$$
\left\|\mathbf{b}_{m}\right\|^{2}=p_{m} \leq P_{\text {max }}, \quad \forall m=1, \ldots, M
$$

and the global power constraint is formulated as follows:

$$
\sum_{m=1}^{M}\left\|\mathbf{b}_{m}\right\|^{2}=\sum_{m=1}^{M} p_{m} \leq M P_{\max }
$$

\subsection{Outage probability constraint}

The outage probability is given by (51). In this subsection, we will reformulate this equation using the beamforming strategy. Proceeding in the same manner as in Subsection 6.2, the outage probability can be written as:

$$
P_{\text {out }}=\operatorname{Prob}\left\{\log _{2}\left(1+\frac{p_{p u} G_{p u}^{2}\left|h_{p u, p u}^{\prime}\right|^{2}}{\sum_{m=1}^{M}\left|\mathbf{h}_{p u, m} \mathbf{h}_{p u, m}{ }^{H}\right|\left|\mathbf{b}_{m}\right|^{2}+\sigma^{2}}\right) \leq R_{p u}\right\}
$$

As in the development of the distributed user selection strategy in Section 6, we introduce here the PU and SU average channel gain estimate $G_{p u}$ and $G_{s u}$, respectively, defined in the Subsection 6.2. These assumptions give the same PU outage probability expression given by (14). 


\subsection{Optimization problem}

Concluding that the maximum eigenvalue $\lambda_{\max }(m)$ must be chosen so as to maximize the capacity of SUs given a fixed transmit power. In the first step of the proposed beamforming user selection strategy, SUs are first pre-selected so as to maximize the per-user sum capacity. In the second step of the user selection strategy, the PU verifies the outage probability constraint and a number of SUs are selected from those pre-selected SUs. If we maximize the per-user sum capacity $\left(C_{s u}\right)$ : i.e. the sum of the SINR averaged over all SUs under the constraints of maintaining the global power lower than $M P_{\max }$ and of satisfying the QoS constraint on outage, the problem can be written as:

$$
\left\{\begin{array}{l}
\text { maximize } f\left(p_{1}, \ldots, p_{M}\right)=\frac{1}{\ln 2} \sum_{m=1}^{M} \ln \left(1+\lambda_{\max }(m) p_{m}\right) \\
\text { subject to } \sum_{m=1}^{M} p_{m} \leq M P_{\max } \\
P_{\text {out }} \leq q
\end{array}\right.
$$

To compute the transmitted power through all SUs, we define the Lagrangian expression for this maximization problem as follows:

$$
J=\frac{1}{\ln 2} \sum_{i=1}^{M} \ln \left(1+\lambda_{\max }(i) p_{i}\right)-\mu\left(\sum_{i=1}^{M} p_{i}-M P_{\max }\right)-v\left(P_{\text {out }}-q\right)
$$

We introduce in (36) two variables, $\mu$ and $v$, called Lagrange multipliers. The solution of all the system is found by calculating the derivatives of $J$ with respect to the power allocation parameters $\left.p_{m}\right|_{m=1 . . M}$ and Lagrange multipliers $\mu$ and $\nu$. By calculating the derivatives of $J$ with respect to the power allocation parameters $p_{m}$, we obtain:

$\frac{\partial J}{\partial p_{m}}=\frac{(\ln 2)^{-1} \lambda_{\max }(m)}{1+\lambda_{\max }(m) p_{m}}-\mu-v \frac{\left(2^{R_{p u}}-1\right) G_{s u}^{2}}{G_{p u}^{2} p_{p u}} \exp \left[-\left(2^{R_{p u}}-1\right)\left(\frac{G_{s u}^{2} \sum_{i=1}^{M} p_{i}+\sigma^{2}}{G_{p u}^{2} p_{p u}}\right)\right]=0$

Let $g\left(p_{i}\right)=\frac{\left(2^{R p u}-1\right) G_{s u}^{2}}{G_{p u}^{2} p_{p u}} \exp \left[-\left(2^{R_{p u}}-1\right)\left(\frac{G_{s u}^{2} \sum_{i=1}^{M} p_{i}+\sigma^{2}}{G_{p u}^{2} p_{p u}}\right)\right]$, we can express the solution of (37) as:

$$
\frac{1}{\left(\mu+v g\left(p_{i}\right)\right) \ln 2} \lambda_{\max }(m)=1+\lambda_{\max }(m) p_{m}
$$


The solution of this problem is formulated as follows:

$$
p_{m}=\frac{1}{\left(\mu+v g\left(p_{i}\right)\right) \ln 2}-\frac{1}{\lambda_{\max }(m)}
$$

The derivatives of $J$ with respect to the power allocation parameters $\left.p_{i}\right|_{i=1 . . M}$ :

$$
\left\{\begin{array}{c}
p_{1}=\frac{1}{\left(\mu+v g\left(p_{i}\right)\right) \ln 2}-\frac{1}{\lambda_{\max }(1)} \\
\cdot \\
p_{M}=\frac{1}{\left(\mu+v g\left(p_{i}\right)\right) \ln 2}-\frac{1}{\lambda_{\max }(M)}
\end{array}\right.
$$

The sum of all equations in (55) gives:

$$
\begin{aligned}
\sum_{i=1}^{M} p_{i} & =\frac{M}{\left(\mu+v g\left(p_{i}\right)\right) \ln 2}-\sum_{i=1}^{M} \frac{1}{\lambda_{\max }(i)} \\
& =M\left(p_{m}+\frac{1}{\lambda_{\max }(m)}\right)-\sum_{i=1}^{M} \frac{1}{\lambda_{\max }(i)} \\
& =M P_{\max }
\end{aligned}
$$

Finally, we obtain the following set of equalities:

$$
p_{m}=P_{\max }-\frac{1}{\lambda_{\max }(m)}+\frac{1}{M} \sum_{i=1}^{M} \frac{1}{\lambda_{\max }(i)} \quad \text { for } m=1, \ldots, M
$$

This equation gives the power allocation solution using the global power constraint given by (33). Firstly, the per-user power constraint given in (32) has been utilized to solve the problem, i.e. maximizing the per-user sum capacity under the constraint of maintaining the per-user power constraint lower than $P_{\max }$ for all users. In this case, the Lagrangian expression is given by:

$$
J=\frac{1}{\ln 2} \sum_{m=1}^{M} \ln \left(1+\lambda_{\max }(i) p_{i}\right)-\sum_{i=1}^{M} \mu_{i}\left(p_{i}-P_{\max }\right)
$$

and the transmitted power through all SUs is:

$$
p_{m}=P_{\max }, \quad m=1, \ldots, M
$$

but it is not the optimal solution. Besides, from (42), $p_{m}$ can have values higher than $P_{\max }$ which contradicts condition (32). To optimally solve this problem, one should adopt this solution:

$$
\begin{array}{ll}
p_{m}=P_{\max } & \text { if } \quad \frac{1}{\lambda_{\max }(m)}<\frac{1}{M} \sum_{i=1}^{M} \frac{1}{\lambda_{\max }(i)} \\
p_{m}=P_{\max }-\frac{1}{\lambda_{\max }(m)}+\frac{1}{M} \sum_{i=1}^{M} \frac{1}{\lambda_{\max }(i)} & \text { else }
\end{array}
$$

Therefore, it will be shown later from simulation results that (45) can approximate very well the per-user sum capacity with optimal power allocation. 


\section{Distributed game theory-based resource allocation strategy}

\subsection{Game theory tools}

Game theory was at first a mathematical tool used for economics, political and business studies. It helps understand situations in which decision-makers interact in a complex environment according to a set of rule (M. J. Osborne, 2003). Many different types of game exist which are used to analyze different situations: potential games, repeated game, cooperative or non-cooperative games. In the cognitive radio network (CRN), the formal game model for the power control can be defined as follows:

- Players: are the cognitive users (secondary users (SUs)).

- Actions: called also as the decisions, and are defined by the transmission power allocation strategy.

- Utility function: represents the value of the observed quality-of-service (QoS) for a player, and is defined later in this section.

The central idea in game theory is how the decision from one player will affects the decision-making process from all other players and how to reach a state of equilibrium that would satisfy most of the players. A well known contributor in the field is Nash for the Nash equilibrium (R. W. Rosenthal, 1973). The theory shows that you can reach a state equilibrium for your system where all decisions are set, unchanging and is the best possible situation for the players.

CR need to perform sophisticated adaptation and dynamically learn from the environment. This situation makes the learning process a very complicated one comparable to situation found in economics. Game theory is already used in other field of communication to better understand congestion control, routing, power control, topology control and trust management (A. B. MacKenzie, L. Dasilva, and W. Tranter, 2006). Our interests rest in its use for power control as it can be considered a game with fixed number of players where each tries to optimize their power levels. There are a number of properties that makes this problem appropriate for a cognitive radio game model:

- The player's payoff is a function of her own transmit power level and her signal-to-noise and interference ratio (SINR). The player's SINR is a function of her own transmit power and the transmit powers of the other players in the cell.

- When a player increases her power level, this will increase her own SINR, but will decrease the SINRs of all other players.

- For a fixed SINR, the players prefer lower power levels to higher ones. That is, players wish to conserve power and extend their battery life when possible.

- For a fixed power level, players prefer higher SINR to lower one. That is, players want the best possible channel conditions for a given expenditure of power.

There are many ways to cope with these issues such as to add restriction to the use of the power resource by charging it to users. This is done by adding a cost component to the payoff function to add fairness to the network. Another idea is to model the scenario as a repeated game (A. B. MacKenzie, L. Dasilva, and W. Tranter, 2006). 
In this part we formulate the problem of resource allocation in the context of a CRN to reflect the needs of PUs and SUs. We consider the primary uplink of a single CRN, where cognitive transmitters transmit signals to a number of SUs, while the primary BS receives its desired signal from a primary transmitter and interference from all the cognitive transmitters.

To resolve the problem of resource allocation, we propose a utility function that meets the objective to maximize the SUs capacity, and the protection for PUs. Specifically, we define a payoff function that represents the SNIR constraint, and a price function specifies the outage probability constraint. The utility function is defined as:

$$
\text { utility function }=\text { payoff function }- \text { price function }
$$

We introduce a payoff to express the capacity need of SU $m$, and a price function to represent the protection for PUs by means of the outage probability. And each SU adjusts its transmitted power to maximize its utility function. Therefore, we will present in this part a power allocation algorithm that maximize the defined utility function to compute the transmitted power of each SU.

\subsection{Power allocation algorithm}

We derive in this section the utility function: we define a payoff function specifies the SU capacity constraint and a price function that represents the interference constraint as a function of the outage probability constraint. Therefore, the price function is given by:

$$
\mathrm{L}_{\mathrm{Intf} m}=\frac{p_{m}\left|h_{p u, m}\right|^{2}}{P_{T}-\sum_{\substack{l=1 \\ l \neq m}}^{M} p_{l}\left|h_{p u, l}\right|^{2}}
$$

which is a normalized value. As long as this ratio $\in[0,1]$, the protection for PU is met. The margin of $P_{T}-\sum_{\substack{l=1 \\ l \neq m}}^{M} p_{l}\left|h_{p u, l}\right|^{2}$ is the maximum interference that SU $m$ could generate. We will derive in this section the equation of the interference constraint $P_{T}$ as a function of the outage probability.

To proceed further with the analysis and for the sake of emphasis, we introduce the PU average channel gain estimate $G_{p u}$ based on the following decomposition:

$$
h_{p u, p u} \equiv G_{p u} * h_{p u, p u}^{\prime}
$$

where $h_{\text {pupu }}^{\prime}$ is the random component of channel gain and represents the normalized channel impulse response tap. This gives us the following PU outage probability expression in an interference-limited context:

$$
P_{\text {out }} \simeq \operatorname{Prob}\left\{\left|h_{p u, p u}^{\prime}\right|^{2} \leq\left(2^{R_{p u}}-1\right)\left(\frac{\sum_{m=1}^{M} p_{m}\left|h_{m, p u}\right|^{2}}{G_{p u}^{2} p_{p u}}\right)\right\}
$$


From now on we assume for simplicity of analysis that the channel gains are i.i.d rayleigh distributed. However, the results can be immediately translated into results for any other channel model by replacing by the appropriate probability distribution function. Continuing from (48), we have:

$$
P_{\text {out }} \simeq \int_{0}\left(2^{R_{p u}}-1\right)\left(\frac{\sum_{m=1}^{M} p_{m}\left|h_{m, p u}\right|^{2}}{G_{p u}^{2} p_{p u}}\right) \exp (-t) d t
$$

Finally, we get the following outage constraint:

$$
P_{\text {out }} \simeq 1-\exp \left[-\left(2^{R_{p u}}-1\right)\left(\frac{\sum_{m=1}^{M} p_{m}\left|h_{m, p u}\right|^{2}}{G_{p u}^{2} p_{p u}}\right)\right]
$$

Replacing the interference constraint equation in (50), we can express the probability outage as:

$$
P_{\text {out }} \simeq 1-\exp \left[-\left(2^{R_{p u}}-1\right) \frac{P_{T}}{G_{p u}^{2} p_{p u}}\right]
$$

Then, the corresponding interference constraint is:

$$
P_{T} \simeq \frac{p_{p u} G_{p u}^{2}}{1-2^{R_{p u}}} \ln \left(1-P_{\text {out }}\right)
$$

We introduce now a utility function for which each SU adjusts its transmitted power in order to maximize it. It is composed of a payoff function expressed as the capacity $C_{m}$ of the SU, and of a price function composed of the interference level to the PU and the power consumption.

Then, the utility function is expressed as follow:

$$
U_{m}=C_{m}-\left(\frac{p_{m}\left|h_{p u, m}\right|^{2}}{P_{T}-\sum_{\substack{l=1 \\ l \neq m}}^{M} p_{l}\left|h_{l, m}\right|^{2}}\right)^{a_{m}}
$$

The parameter $a_{m}$ is adjustable to have a comparable values, i.e. the payoff function value and the price function value. This parameter gives the flexibility needed to adjust the SU capacity over the interference to the PU. We choose $a_{m}<0$. It could be easily obtained that the price function decreases as the ratio $\mathrm{L}_{\mathrm{Intf} m}$ increases. This fact is caused by the negative property of $a_{m}$. 
Mathematically, the game $G$ can be expressed as:

$$
\text { Find }\left.p_{m}\right|_{m=1, \ldots, M}=\arg \max _{p_{m}} U_{m}\left(p_{m}, \mathbf{P}_{-m}\right)
$$

subject to:

$$
\left\{\begin{array}{l}
\sum_{m=1}^{M} p_{m}\left|h_{p u, m}\right|^{2} \leq P_{T} \\
P_{\text {out }} \leq q \\
0 \leq p_{m} \leq P_{\text {max }}
\end{array}\right.
$$

Recall that $p_{m}$ denotes the strategy adopted by SU $m$ and $\mathbf{P}_{-m}=\left(p_{l}\right)_{l \neq m, l \in\{1, \ldots, M\}}$ denotes the strategy adopted by the other SUs. We replace the capacity by expression given by (4) and use (9) to obtain the following equation:

$$
U_{m}=\log _{2}\left(1+\operatorname{SINR}_{m}\right)-\left(\frac{\left|h_{p u, m}\right|^{2}}{P_{T}-\sum_{\substack{l=1 \\ l \neq m}}^{M} p_{l}\left|h_{l, m}\right|^{2}}\right)^{a_{m}} \times\left(\frac{\operatorname{SINR}_{m} \operatorname{Intf}_{m}}{\left|h_{m, m}\right|^{2}}\right)^{a_{m}}
$$

We are going to maximize the utility function in terms of the SINR. The solution of the system is found by calculating the derivatives of $U_{m}$ with respect to the signal-to-noise and interference ratio parameters $\operatorname{SINR}_{m}$ :

$$
\frac{\partial U_{m}}{\partial \operatorname{SINR}_{m}}=\frac{1}{\overline{\left(1+\operatorname{SINR}_{m}\right) \ln 2}}-\left(\frac{\left|h_{p u, m}\right|^{2}}{P_{T}-\sum_{\substack{l=1 \\ l \neq m}}^{M} p_{l} h_{l, m}}\right)^{a_{m}} \times a_{m}\left(\frac{\operatorname{SINR}_{m} \operatorname{Intf}_{m}}{\left|h_{m, m}\right|^{2}}\right)^{a_{m}-1} \frac{\operatorname{Intf}_{m}}{\left|h_{m, m}\right|^{2}}
$$

We can express the solution of (57) as:

$$
\left(1+\operatorname{SINR}_{m}\right) \operatorname{SINR}_{m}^{a_{m}-1}=\frac{1}{a_{m} \beta_{m} \ln 2}
$$

where:

$$
\beta_{m}=\left(\frac{\left|h_{p u, m}\right|^{2}}{P_{T}-\sum_{\substack{l=1 \\ l \neq m}}^{M} p_{l}\left|h_{l, m}\right|^{2}}\right)^{a_{m}}\left(\frac{\operatorname{Intf}_{m}}{\left|h_{m, m}\right|^{2}}\right)^{a_{m}}
$$


denoting the slope of the price function. Let $f\left(\operatorname{SINR}_{m}\right)=\left(1+\operatorname{SINR}_{m}\right) \operatorname{SINR}_{m}^{a_{m}-1}$. Finally, we obtain the following set of equalities:

$$
\mathrm{SINR}_{m}=f^{-1}\left(\frac{1}{a_{m} \beta_{m} \ln 2}\right)
$$

The maximization problem is dependent on $a_{m}$ which is defined in the utility function as an adjustment parameter to the price function. For simulation results $a_{m}=-0.2$. It was chosen to stay with this value after different simulations to show its influence on the obtained results.

\subsection{Existence and uniqueness of the Nash equilibrium}

In the proposed game, each SU chooses an appropriate power to maximize its utility function. In this context, it is important to ensure the stability of the system. A concept which relates to this issue is the Nash equilibrium. As definition in (R. W. Rosenthal, 1973), a pure strategy profile $\left\{p_{l}^{*}\right\}_{l \neq m, l \in\{1, \ldots, M\}}$ is a Nash equilibrium of the proposed game if, for every player $m$ (i.e. $\mathrm{SU} m$ ):

$$
U_{m}\left(p_{m}^{*}, \mathbf{P}_{-m}^{*}\right) \geq U_{m}\left(p_{m}, \mathbf{P}_{-m}^{*}\right), \quad \forall m \in\{1, \ldots, M\}
$$

A Nash equilibrium can be regraded as a stable solution, at which none of the users has the incentive to change its power $p_{m}$.

\subsubsection{Existence of the Nash equilibrium}

Theorem 1: Game G admits at least one Nash equilibrium.

proof: The conditions for the existence of Nash equilibrium in a strategic game are given in (Fudenberg \& Tirole, 1991):

1. The set $P_{m}$ is a nonempty, convex, and compact subset of some Euclidean space for all $m$.

2. The utility function $U_{m}\left(p_{m}, \mathbf{P}_{-m}\right)$ is continuous on $P$ and quasi-concave on $P_{m}$.

According to the above description of the strategy space, it is straightforward to see that $P_{m}$ is nonempty, convex and compact. Notice that $U_{m}\left(p_{m}, \mathbf{P}_{-m}\right)$ is a linear function of either $p_{m}$, which means the second condition is satisfied. Hence, game G admits at least one Nash equilibrium.

\subsubsection{Uniqueness of the Nash equilibrium}

Theorem 2: Game $G$ always possesses a unique Nash equilibrium under the sufficient conditions.

proof: It's established in (Yates, 1995) that if the utility function $U_{m}\left(p_{m}\right):\left(p_{m}\right)_{m \in\{1, \ldots, M\}}$ is a standard function, then the Nash equilibrium in this game will be unique. A function $f(x)$ is said to be a standard function if it satisfies the following three properties (Yates, 1995):

1. Positivity: $f(x)>0$.

2. Monotonicity: If $x \geq x^{\prime}$, then $f(x) \geq f\left(x^{\prime}\right)$.

3. Scalability: For all $\mu>1, \mu f(x) \geq f(\mu x)$. 
The positivity is obviously satisfied by adjusting parameter $a_{m}$.

Considering $p_{m} \geq p_{m}^{\prime}$, we have

$$
C_{m}\left(p_{m}\right) \geq C_{m}\left(p_{m}^{\prime}\right)
$$

Using the propriety that $a_{m}<0$, we can obtain that

$$
\left(\frac{p_{m}\left|h_{p u, m}\right|^{2}}{P_{T}-\sum_{\substack{l=1 \\ l \neq m}}^{M} p_{l}\left|h_{l, m}\right|^{2}}\right)^{a_{m}} \leq\left(\frac{p_{m}^{\prime}\left|h_{p u, m}\right|^{2}}{P_{T}-\sum_{\substack{l=1 \\ l \neq m}}^{M} p_{l}\left|h_{l, m}\right|^{2}}\right)^{a_{m}}
$$

According to (62) and (63), the monotonicity property is proved $\forall m \in\{1, \ldots, M\}$.

For all $\mu>1$, it's got that:

$$
\mu C_{m}\left(p_{m}\right)=\mu \log _{2}\left(1+\operatorname{SINR}_{m}\right)=\log _{2}\left(1+\operatorname{SINR}_{m}\right)^{\mu} \geq \log _{2}\left(1+\mu \operatorname{SINR}_{m}\right)=C_{m}\left(\mu p_{m} \chi 64\right)
$$

Since $a_{m}<0$, we have also:

$$
\left(\frac{\mu p_{m}\left|h_{p u, m}\right|^{2}}{P_{T}-\sum_{\substack{l=1 \\ l \neq m}}^{M} p_{l}\left|h_{l, m}\right|^{2}}\right)^{a_{m}} \mu^{a_{m}}\left(\frac{p_{m}\left|h_{p u, m}\right|^{2}}{P_{T}-\sum_{\substack{l=1 \\ l \neq m}}^{M} p_{l}\left|h_{l, m}\right|^{2}}\right)^{a_{m}} \leq \mu\left(\frac{p_{m}\left|h_{p u, m}\right|^{2}}{P_{T}-\sum_{\substack{l=1 \\ l \neq m}}^{M} p_{l}\left|h_{l, m}\right|^{2}}\right)^{a_{m}}
$$

Finally, according to (64) and (65) the scalability property is proved. Therefore, the proposed game $G$ always possesses a unique Nash equilibrium.

\section{Performance evaluation}

To go further with the analysis, we resort to realistic network simulations. Specifically, we consider a CRN in the downlink and the uplink mode, respectively, with one PU and $M$ SUs attempting to communicate during a transmission, subject to mutual interference. A hexagonal cellular system functioning at $1.8 \mathrm{GHz}$ with a secondary cell of radius $R$ and a primary protection area of radius $R_{p}$ is considered. Secondary transmitters may communicate with their respective receivers of distances $d<R_{p}$ from the BS. We assume that the PU and the SUs are randomly distributed in a two-dimensional plane.

The channel gains are based on the COST-231 Hata model (Urban Transmission Loss Models for Mobile Radio in the 900 and $1800 \mathrm{MHz}$ Bands, 1991) including log-normal shadowing with standard deviation of $10 \mathrm{~dB}$, plus fast-fading assumed to be i.i.d. circularly symmetric with distribution $\mathcal{C N}(0,1)$.

The performance of the proposed distributed user selection strategy is evaluated by Monte Carlo simulations $\left(I T_{\max }=10^{4}\right)$. It is assumed that the maximum outage probability $q=1 \%$ 


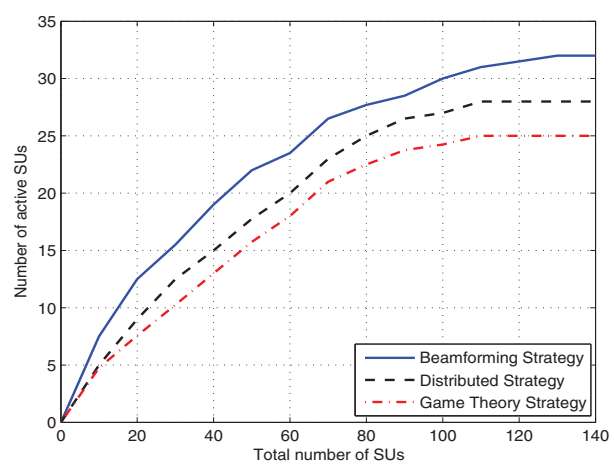

(a) Downlink

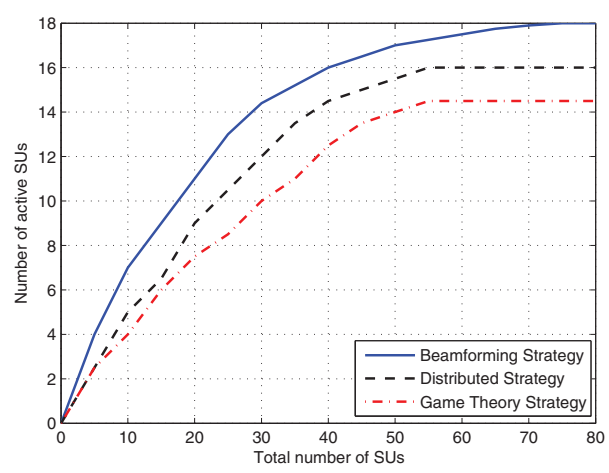

(b) Uplink

Fig. 3. Performance evaluation of the proposed user selection strategies: Number of active secondary users versus total number of secondary users with rate $=0.1 \mathrm{bits} / \mathrm{s} / \mathrm{Hz}$ and $q=1 \%$ in the downlink and the uplink mode.

for both distributed and centralized algorithms. We considered also that the radius of the secondary cell $R=1000$ meters and the radius of the primary protection area $R_{p}=600$ meters. The derivation of the maximum number of SUs allowed to transmit using the distributed algorithm is based on the average channel gains $G_{s u}$ and $G_{p u}$ estimation. From the locations of the users in the two-dimensional plane and the propagation characteristics of the environment, we can estimate the two average channel gains for the downlink and the uplink mode. These values are estimated assuming a wireless ad hoc network affected by a large number of interferers. From simulation results, using $M=500$ SUs and one PU, we find $G_{p u}^{2} / G_{s u}^{2} \simeq 15$ in the downlink mode and $G_{p u}^{2} / G_{s u}^{2} \simeq 20$ in the uplink mode.

Fig. 3 shows the behavior of the three strategies, for both downlink and uplink scenarios. This figure presents the number of active SUs versus the total number of SUs ranging between 1 user and a maximum of 140 users, and using a rate equal to $0.1 \mathrm{bits} / \mathrm{s} / \mathrm{Hz}$ ). It can be seen from the figure that increasing the number of SUs produces improvements in the number of active SUs. We show also that the beamforming user selection method outperforms the game theory-based and the distributed strategy. We gain almost 4 additional active SUs using the centralized beamforming strategy in comparison with the simple distributed strategy. It is obvious from Fig. 3 that the number of active SUs slopes in the distributed strategy start dropping at a lower number of SUs than in the beamforming strategy case. While the number of active SUs curve has dropped off starting from approximately 40 SUs in the distributed algorithm, the curve of the beamforming strategy starts dropping off after 60 SUs for the beamforming strategy, in the case of downlink scenario. Observing this figure, we get also that the number of active SUs is lower than 20 users for any number of transmitter SUs in the system. This means that the PU outage probability is upper-bounded by the maximum outage probability $q$. Fig. 4 confirms these results. As reflected in the figure, the required maximum outage probability is respected, since all outage probability values are lower then $1 \%$ for any number of SUs. From these results, the $P_{\text {out }}$ curves in both uplink and downlink cases can be observed to have very similar slopes as in Fig. 3. 


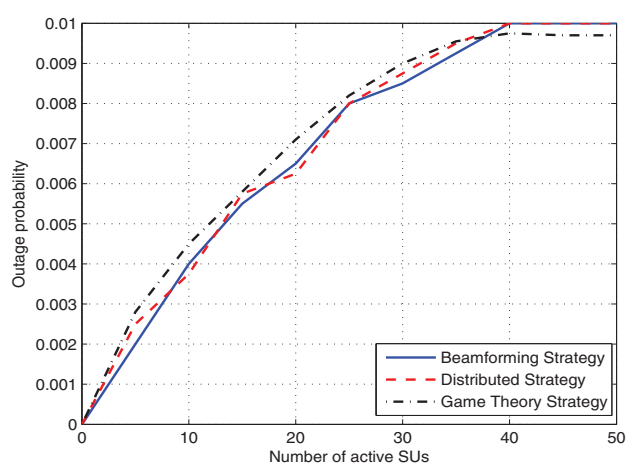

(a) Downlink

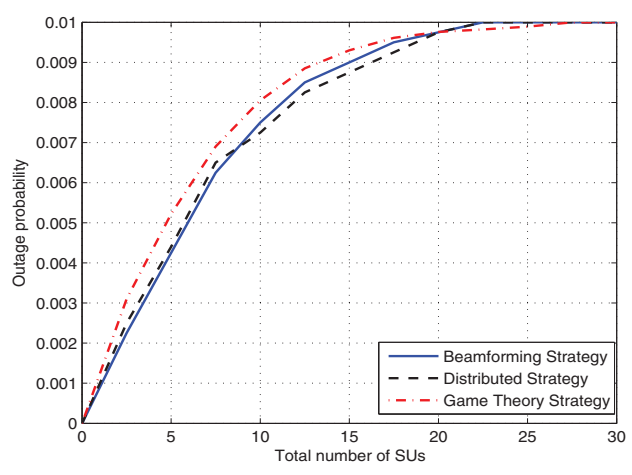

(b) Uplink

Fig. 4. Performance evaluation of the proposed user selection strategies: Outage probability as function of the number of secondary users for a target outage probability $=1 \%$ and a rate $=0.3 \mathrm{bits} / \mathrm{s} / \mathrm{Hz}$ in the downlink and the uplink mode.

So far, we verified the first goal of the proposed methods, maintaining the outage probability of the PU not degraded. The second goal in developing these new strategies and specially the beamforming-based one is to reduce the interference from SUs transmitters. However, we must show the impact of the proposed centralized beamforming scheme on the interference power. Fig. 5 depicts the normalized interference power of the beamforming user selection strategy versus the number of SUs in the uplink mode, in comparison with the distributed and the centralized methods. This figure shows that the interference power increases with the increasing number of SUs. It shows as well that the beamforming strategy performs better in terms of interference power. On the other hand, the distributed and the centralized strategies have virtually identical curves. Indeed, the proposed technique reduces interfering power by about $45 \%$ in comparison with the distributed and the centralized techniques. Therefore, we conclude that the beamforming strategy is highly efficient in terms of reducing the interference power as well as robust in maintaining a certain QoS to a PU.

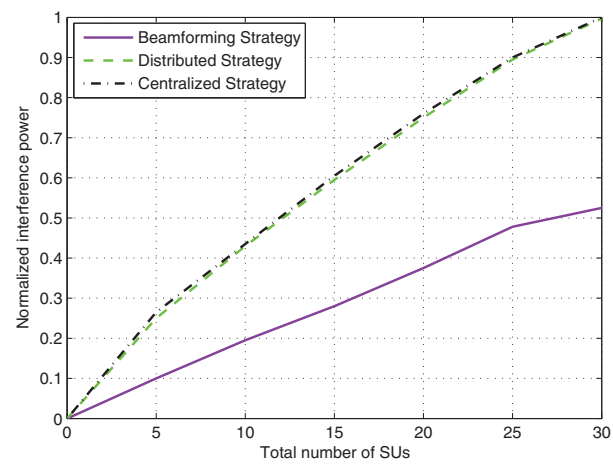

Fig. 5. Performance evaluation of the user selection strategies: Interference power versus number of SUs with $q=1 \%$ and a rate $=0.3 \mathrm{bits} / \mathrm{s} / \mathrm{Hz}$ in the uplink mode. 


\section{Conclusion}

In this chapter, we focused on resource allocation and interference management. Within this setting, we considered different system models in which SUs compete for a chance to transmit simultaneously or orthogonally with the PU. On the basis of these models, we have also defined the specific resource allocation problem and offer insights into how to design such scenario in a CRN environments and we proposed three user selection strategies. One first key idea is based on outage probability to mange the QoS of the CR system. We have derived a distributed user selection algorithm under a cognitive capacity maximization criterion and outage probability constraint. We found out that we should make a tradeoff between cognitive capacity maximization and number of active SUs maximization. Then, we investigated the problem of resource allocation for multiuser multi-antenna channels using a beamforming strategy. The proposed strategy was proved to be the optimal one that achieves the maximum rate for both users under the constraint that the SU guarantees a QoS for the primary system within the outage probability constraint. We have explicitly derived the capacity of the primary as well as the SU. Finally, we explored the idea of combining game theory with resource allocation in CRN. Specifically, we defined a utility/pricing strategy that meets the objective to maximize the SUs capacity and the protection for PUs. Indeed, we discussed the existence of the Nash equilibrium of the proposed game, as well as its uniqueness. We demonstrated that the proposed game admits one and only one Nash equilibrium. Both theoretical and simulation results based on a realistic network setting provide substantial throughput gains, thereby illustrating interesting features in terms of CRN deployment while maintaining QoS for the primary system by means of outage probability for the three strategies. The main contribution within this work is the QoS management of the CR system. The originality in the proposed methods is that we guarantee a QoS to PU by maintaining the PU's outage probability unaffected in addition to a certain QoS to SUs and ensuring the continuity of service even when the spectrum sub-bands change from vacant to occupied. Thus by the outage probability control, if we have a vacant spectrum holes in the PU band, we set the outage probability $P_{\text {out }}=1$ to exploit the available spectrum band by SUs, and if we have occupied sub-bands, the outage probability is set to $P_{\text {out }}=q$ depending on the PU's QoS.

\section{Acknowledgements}

The research leading to these results has received funding from the European Community's Seventh Framework Programme (FP7/2007-2013) under grant agreement SACRA n²49060. This work was supported also by the European projects: ACROPOLIS (advanced coexistence technologies for radio optimization and unlicensed spectrum), and CROWN (cognitive radio oriented wireless networks).

\section{References}

A. B. MacKenzie, L. Dasilva, and W. Tranter (2006). Game theory for wireless engineers, Morgan and Claypool Publishers .

A. Gjendemsjø, D. Gesbert, G. E. Øien and S.G. Kiani (2007). Binary power control for multi-cell capacity maximization, IEEE Trans. Wireless Comm. . 
A. Tarighat, M. Sadek and A.H. Sayed (2005). A multi user beamforming scheme for downlink mimo channels based on maximizing signal-to-leakage ratios, IEEE International Conference on Acoustics, Speech, and Signal Processing, ICASSP .

B. Zayen, A. Hayar and G. Noubir (2011). Utility/pricing-based resource allocation strategy for cognitive radio systems, ICMCS'11, 2nd International Conference on Multimedia Computing and Systems .

B. Zayen, A. Hayar and G.E. Øien (2009). Resource allocation for cognitive radio networks with a beamforming user selection strategy, Asilomar .

B. Zayen, M. Haddad, A. Hayar and G.E. Øien (2008). Binary power allocation for cognitive radio networks with centralized and distributed user selection strategies, Elsevier Physical Communication Journal 1(3): 183-193.

D. Schmidt, C. Shi, R. Berry, M. Honig and W. Utschick (2009). Distributed resource allocation schemes, IEEE Signal Process. Mag. 26(5): 53-63.

E. Jorswieck and R. Mochaourab (2010). Beamforming in underlay cognitive radio: Null-shaping design for efficient nash equilibrium, International Workshop on Cognitive Information Processing.

E.A. Jorswieck and H. Boche (2004). Channel capacity and capacity-range of beamforming in mimo wireless systems under correlated fading with covariance feedback, IEEE Transactions on Wireless Communications 3(5).

F. Wang, M. Krunz and S. Cui (2008). Spectrum sharing in cognitive radio networks, IEEE Conference on Computer Communications, pp. 1885-1893.

FCC (2003). Cognitive radio technologies proceeding, Web Page, http://www.fcc.gov/oet/cognitiveradio/.

Fudenberg, D. \& Tirole, J. (1991). Game Theory, MIT Press, Cambridge,MA.

J. Huang, R.A. Berry and M.L. Honig (2006). Auction-based spectrum sharing, Mobile Networks and Applications, Vol. 11, pp. 405-418.

J. Huang, Z. Han, M. Chiang and V. Poor (2007). Auction-based distributed resource allocation for cooperation transmission in wireless networks, IEEE Global Communications Conference (GLOBECOM).

J. Mitola (1999). Cognitive radio for flexible mobile multimedia communications, Mobile Multimedia Communications (MoMUC), New York.

J. S. Pang, G. Scutari, D. Palomar and F. Facchinei (2010). Design of cognitive radio systems under temperature-interference constraints: A variational inequality approach, IEEE Trans. Signal Process. 58(6): 3251-3271.

Kiani, S. G. \& Gesbert, D. (2006). Optimal and distributed scheduling for multicell capacity maximization. IEEE International Conference on Communications.

L. Akter and B. Natarajan (2009). Qos constrained resource allocation to secondary users in cognitive radio networks, Elsevier, Computer Communications .

L. H. Ozarow, S. Shamai and A.D. Wyner (1994). Information theoretic considerations for cellular mobile radio, IEEE Trans. Veh. Technol. 43(5): 359-378.

L. Qian, X. Li, J. Attia and Z. Gajic (2007). Power control for cognitive radio ad hoc networks, IEEE Workshop on Local and Metropolitan Area Networks, pp. 07-12.

M. Haddad, A. Hayar and G.E. Øien (2008). Uplink distributed binary power allocation for cognitive radio networks, CrownCom, Singapore.

M. J. Osborne (2003). An introduction to game theory, Oxford University Press . 
M. Schubert and H. Boche (2004). Solution of the multiuser downlink beamforming problem with individual sinr constraints, IEEE Trans. Veh. Technol. 53(1): 18-28.

N. Nie and C. Comaniciu (2005). Adaptive channel allocation spectrum etiquette for cognitive radio networks, New Frontiers in Dynamic Spectrum Access Networks.

P. Cheng, Z. Zhang, H. Huang and P. Qiu (2008). A distributed algorithm for optimal resource allocation in cognitive ofdma systems, IEEE International Conference on Communications.

P. Viswanath, D. Tse and R. Laroia (2004). Random beamforming for mimo systems with multiuser diversity, IEEE Personal, Indoor and Mobile Radio Commun. Conf. 1: 290-294.

Peha, J. M. (2005). Approaches to spectrum sharing, IEEE Communications Magazine 43(2): 10-12.

R. W. Rosenthal (1973). A class of games possessing pure-strategy nash equilibria, International Journal of Game Theory 2 pp. 65-67.

Tse, D. (2004). Fundamentals of Wireless Communication, University of California, Berkeley Pramod Viswanath, University of Illinois, Urbana-Champaign.

Urban Transmission Loss Models for Mobile Radio in the 900 and $1800 \mathrm{MHz}$ Bands (1991).

Y. Wu and D.H.K. Tsang (2009). Distributed power allocation algorithm for spectrum sharing cognitive radio networks with qos guarantee, IEEE INFOCOM.

Y. Xing, C.N. Mathur, M.A. Haleem, R. Chandramouli and K.P. Subbalakshmi (2007). Dynamic spectrum access with qos and interference temperature constraints, IEEE Transactions on Mobile Computing 6: 423-433.

Yates, R. D. (1995). A framework for uplink power control in cellular radio systems, 13: 1341-1347. 


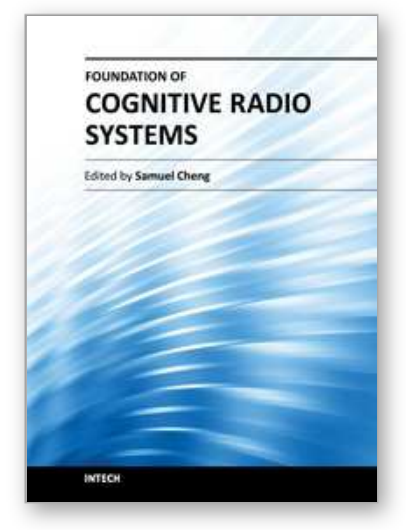

\author{
Foundation of Cognitive Radio Systems \\ Edited by Prof. Samuel Cheng
}

ISBN 978-953-51-0268-7

Hard cover, 298 pages

Publisher InTech

Published online 16, March, 2012

Published in print edition March, 2012

The fast user growth in wireless communications has created significant demands for new wireless services in both the licensed and unlicensed frequency spectra. Since many spectra are not fully utilized most of the time, cognitive radio, as a form of spectrum reuse, can be an effective means to significantly boost communications resources. Since its introduction in late last century, cognitive radio has attracted wide attention from academics to industry. Despite the efforts from the research community, there are still many issues of applying it in practice. This books is an attempt to cover some of the open issues across the area and introduce some insight to many of the problems. It contains thirteen chapters written by experts across the globe covering topics including spectrum sensing fundamental, cooperative sensing, spectrum management, and interaction among users.

\title{
How to reference
}

In order to correctly reference this scholarly work, feel free to copy and paste the following:

Bassem Zayen and Aawatif Hayar (2012). Primary Outage-Based Resource Allocation Strategies, Foundation of Cognitive Radio Systems, Prof. Samuel Cheng (Ed.), ISBN: 978-953-51-0268-7, InTech, Available from: http://www.intechopen.com/books/foundation-of-cognitive-radio-systems/primary-outage-based-resourceallocation-strategies

\section{INTECH}

open science | open minds

\author{
InTech Europe \\ University Campus STeP Ri \\ Slavka Krautzeka 83/A \\ 51000 Rijeka, Croatia \\ Phone: +385 (51) 770447 \\ Fax: +385 (51) 686166 \\ www.intechopen.com
}

\author{
InTech China \\ Unit 405, Office Block, Hotel Equatorial Shanghai \\ No.65, Yan An Road (West), Shanghai, 200040, China \\ 中国上海市延安西路65号上海国际贵都大饭店办公楼 405 单元 \\ Phone: +86-21-62489820 \\ Fax: +86-21-62489821
}


(C) 2012 The Author(s). Licensee IntechOpen. This is an open access article distributed under the terms of the Creative Commons Attribution 3.0 License, which permits unrestricted use, distribution, and reproduction in any medium, provided the original work is properly cited. 